\title{
The Physics of Clusters in Real Gases
}

\author{
By Boris Sedunov*
}

\begin{abstract}
The physics of real gases is interesting both for science and practice. For scientists it is important to move forward from the ideal gas model and to include in the thermal physics of gases the characteristics of molecular interactions. The practitioners need in advanced models of real gases to predict with greater precision their practically valuable properties. The universal laws, such as the van der Waals or corresponding states laws cannot satisfy constantly growing requirements to precision of models for technological gases. The detailed individual characteristics of molecular interactions in gases should be taken into account. But the molecular interactions are shadowed by the thermal movement that complicates their determination. The paper demonstrates the power of the original computer aided analysis of precise experimental thermophysical data contained in modern databases, such as the NIST Thermophysical Properties for Fluid Systems Database. The analysis discovers the cluster structure of pure real gases and the structural transition between different isomers of clusters, provides knowledge of their bond parameters, and opens new features of molecular interactions in gases. The analysis is based on the new variable - the monomer fraction density $D_{m}$, which develops further the fugacity concept. The series expansion coefficients of thermophysical values by powers of $D_{m}$ reflect the cluster fractions' properties and provide the knowledge of their equilibrium constants, bond energies and entropic factors of bound states. The found equilibrium constants for isomers of clusters, like equilibrium constants for chemical compounds, obey to the Boltzmann law. The developed method describes clearly the pair interactions in the dimer fraction of pure real gases and creates the basis to move step-by-step further to trimer and tetramer fractions. For larger numbers of particles in clusters there is a possibility to estimate some averaged characteristics of cluster fractions. This approach helps also to understand better the structure and properties of supercritical fluids.
\end{abstract}

Keywords: Real gas, fluid, chemical equilibrium, thermophysical property, molecular interaction, cluster, dimer, bond energy, equilibrium constant.

\section{Introduction}

The ideal gas model, widely used in science, education and practice, is simple and obvious, but stays rather far from reality, especially for dense gases. It does not take into account the intermolecular forces, discussed by Rowlinson (2002), which are responsible for condensation of matter. The initial step of condensation is the formation of clusters in real gases, analyzed by Feynman (1972), relatively stable complexes of molecules joined together by molecular attractions. The presence of clusters strongly influences properties of real gases. The knowledge of their bond parameters and equilibrium populations is important to make prediction of thermophysical properties for gases with a due

${ }^{*}$ Professor, Russian New University, Russia. 
precision. By turning this interdependence backwards we can extract from experimental thermophysical properties important features of the molecular interaction mechanism basing on the computer aided analysis of precise equilibrium thermophysical data, developed by Sedunov (2013a).

If we ignore the attractions and idealize the repulsions, all gases in equilibrium demonstrate a universal behavior, reflected in the Ideal Gas Law:

$$
\mathrm{P}=\mathrm{RTD} \text {, }
$$

where $\mathrm{P}$ is the pressure, $\mathrm{T}$ - temperature, $\mathrm{D}$ - molar density and $\mathrm{R}$ - universal gas constant. But the individuality of quantum molecular structures and electron density distributions in molecules makes molecular interactions individual for every gas. The deeper we penetrate in molecular interactions the larger becomes an influence of individual characteristics of gases. And thermophysical properties of real gases, described by Poling, Prausnitz and O'Connell (2001), reflect this individuality and are very sensitive to variations of molecular interaction parameters.

Sedunov (2012a) stated that the spectrum of bound states in clusters is shadowed by the thermal movement. It complicates the utilization of spectral analysis to estimate the clusters' bond energies and equilibrium populations. Unlike chemical compounds, where the spectral analysis is very informative, the clusters' properties can be estimated only via processing of experimental thermophysical data. It is important not only for gases, but also for fluids.

The local density in fluids is highly heterogeneous, Table 1. The structural features of clusters in gaseous fluids are reflected in the pore structure of denser fluids. So, the physics of clusters gives the keys to the physics of dense fluids.

Table 1. The Symmetry of Heterogeneities in Fluids

\begin{tabular}{|c|c|c|}
\hline Types of heterogeneity & $\begin{array}{c}\text { Gaseous and gas-like } \\
\text { structures }\end{array}$ & $\begin{array}{c}\text { Liquid and liquid-like } \\
\text { structures }\end{array}$ \\
\hline $\begin{array}{c}\text { Nanosized objects in } \\
\text { fluids, which densities are } \\
\text { far from critical }\end{array}$ & $\begin{array}{c}\text { Low density real gases } \\
\text { with small clusters }\end{array}$ & $\begin{array}{c}\text { Dense liquids with point } \\
\text { vacancies and small } \\
\text { pores }\end{array}$ \\
\hline $\begin{array}{c}\text { Macroscopic } \\
\text { heterogeneous objects near } \\
\text { critical density }\end{array}$ & $\begin{array}{c}\text { Fog-like structure with } \\
\text { drop-like clusters }\end{array}$ & $\begin{array}{c}\text { Foam-like structure with } \\
\text { bubble-like pores }\end{array}$ \\
\hline
\end{tabular}

Currently there are two opposite approaches to the problem of molecular interactions and clusters in equilibrium real gases:

- The molecular dynamics (MD) and model potentials argued by Kaplan (2006).

- The cluster fractions' properties extraction from precise experimental thermophysical data suggested by Sedunov (2012b, 2013a).

A widely used approach to molecular interactions is based on model potentials. The MD methods to provide a satisfactory correspondence to 
experimental data need in better knowledge of the molecular interaction mechanisms than the widely used simplified models, like the suggested by Lennard-Jones (1924) interaction potential. The interaction mechanisms are much more complicated and cannot be reduced to a system of central forces acting between centers of molecules. At collisions molecules are subject to huge forces that excite their rotations and vibrations and also change their electron density distributions. The excitations during the particle flight in the attraction zone may be responsible for the excess centripetal kinetic energy redistribution between internal molecular degrees of freedom. Without this redistribution the colliding particles cannot convert their infinite mode of movement into the finite mode inherent to clusters. The simplification of molecular interaction mechanisms in MD simulations does not permit to estimate thermophysical properties at a level of experimental data precision.

So, we should first improve our knowledge of the molecular interaction mechanisms and parameters basing on precise experimental data. The extraction of cluster bond parameters should be based on the most precise experimental data and the most effective algorithms of data processing. We have selected the step-by-step approach from the simplest pair interactions in dimers to larger clusters. The larger is the number of particles in the cluster, the greater is the influence of experimental errors on precision of the cluster bond parameters evaluation.

For liquids and liquid mixtures the Statistical Associating Fluid Theory (SAFT), suggested by Economou (2002) and developed by Kontogeorgis and Folas (2009), provides interesting and practically valuable results. It is based on experimental thermophysical data for dense fluids. In this aspect our approach stays close to this well known theory. There is a challenging task to join the SAFT achievements and the physics of clusters to be discussed here.

The goal of this paper is to demonstrate the computer aided analysis of precise thermophysical data for different pure gases, including the method and results: the clusters' bond parameters and the transitions between different isomer configurations of clusters at a changing temperature. But for this analysis it was needed to find the most informative thermophysical properties and variables and the most reliable data.

\section{Why the Investigation of Clusters is Important:}

- The clusters are an initial step in condensation of Matter!

- They are the first complex objects formed in gaseous clouds of young Universe;

- The clusters are as multiple as chemical compounds, but their bond energies are much lower than the chemical energies and sometimes lower than the ambient temperature;

- Nevertheless, a strong influence of clusters on our life and modern technologies motivates us to study them better!

The Problem of Clusters Investigation: 
- The energies of bound states in clusters are smashed by thermal movement that makes ineffective the spectroscopy;

- For this reason we know Galaxies and elementary particles better than the clusters;

- The determination of the cluster bond parameters requires the highest precision of experimental data and sophisticated methods of data processing.

Original Points of this Research:

- Computer aided method of the molecular interaction and cluster bond parameters extraction from precise thermophysical data;

- Monomer fraction density (MFD) as the main variable in the theory of clusters in equilibrium real gases;

- Potential energy as the most informative thermophysical property in the physics of clusters;

- Series expansion of the potential energy density by powers of the monomer fraction density as the source of information about properties of clusters and cluster fractions in equilibrium real gases;

- Taking into account the monomer-monomer repulsion energy in computations of the dimer fraction properties;

- Cluster bond energy definition, like the dissociation energy for a chemical compound, as an averaged energy for its decomposition to monomers;

- The principle of the cluster bond energy flattening at high temperatures;

- Potential energy based equilibrium constants for cluster fractions;

- Soft structural transition between isomer modifications of clusters;

- The cluster equilibrium constant correspondence to the Boltzmann law at temperatures with domination of only one isomer.

\section{The Computer Aided Analysis of Experimental Thermophysical Data}

\section{The Sources of Experimental Data for the Analysis}

In modern science for many pure gases due to collective efforts of researchers from all over the World a comprehensive set of experimental data for equilibrium thermophysical properties has been accumulated. But raw experimental data are not precise enough to be used in the analysis aimed at the extraction of molecular interaction parameters. The parameters extraction task is related to inverse mathematical problems, argued by Aster, Borchers and Thurber (2012), the results of which diverge at smallest fluctuations of initial data. To escape from this divergence we should base our analysis on regularized initial data. The regularization is now performed by TRC.NIST (2014) during creation of electronic databases, like the online 
WEBBOOK.NIST (2011) “Thermophysical Properties of Fluid Systems". All initial data for this research have been taken from this Database. The regularization of data includes the critical evaluation of experimental data and their interpolation by high order polynomials accounting for the thermodynamics based correlations between thermophysical properties, described by Landau and Lifshits (1980).

The developed by author thermal analysis of regularized experimental data method provides a physically clear vision of the cluster fractions' structure in pure real gases. The method is very sensitive to precision of experimental data in databases. It feels the difference of the universal gas constant values for data tables, which have been created in different periods of history. For this reason instead of the contemporary nominal $\mathrm{R}$ value for the data processing Sedunov (2013b) suggested to estimate the corresponding effective value $R_{\text {ef }}$ as the zero pressure limit of the P/TD ratio. For every gas it is needed to estimate its own $\mathrm{R}_{\mathrm{ef}}$ value. We hope that with time the data in the NIST database may be transformed to the unique universal gas constant, but now the data reflect the history of their creation and correspond to different $\mathrm{R}_{\mathrm{ef}}$.

In this research the most reliable results have been received for small equilibrium clusters with numbers of particles from two to four. For larger number of particles the results become less reliable. But the reproducibility of the cluster fractions' properties for small clusters is itself a remarkable result. It witnesses the correctness of used regularized experimental data and discovers new features of molecular interactions.

\section{The Monomer Fraction Density as the Main Variable for Thermophysical Data} Analysis

The monomer fraction density, introduced by Sedunov (2008) in a family of thermodynamics variables, provides the discovery of cluster structures and properties via the computer aided analysis of precise thermophysical data.

Numerous attempts to find properties of clusters via virial expansion of thermophysical data, argued by Mayer and Goeppert-Mayer (1977), failed, as it was stated by Feynman (1972). The virial expansions are very effective in the regularization of raw experimental data, but their coefficients are combinations of contributions from different cluster fractions, because the used in virial expansions arguments, such as a total density or a total pressure, are sums of partial values for different cluster fractions.

The Law of Mass Action for chemical equilibrium media, argued by Koudryavtsev, Jameson and Linert (2001), states that the equilibrium concentrations of compounds depend on the concentrations of reacting elementary components raised to the corresponding powers. Similarly, to find the equilibrium concentration of a cluster fraction we should raise the concentration of monomers to the number of basic particles in the cluster. Therefore, instead of the total molar density, we should use the molar density of monomers as an argument for series expansion of a thermophysical value to find the partial contribution of a corresponding cluster fraction in this value. 


\section{Monomers in Real Gases}

Real gases and denser fluids are composed of basic particles that determine the chemical nature of the fluid. For example, $\mathrm{Ar}$ atoms or $\mathrm{N}_{2}$ molecules are basic particles for Argon or Nitrogen real gases. But in real gases the basic particles form agglomerations, named clusters. The basic particles not entering the clusters, named monomers, are elementary components of a real gas. They temporarily fly far from other particles without noticeable interaction with them. It is difficult to define the difference between monomers and bound particles basing on the MD approach. But the phenomenological approach based on thermodynamics gives a correct determination of the monomer fraction density in spite of the essential heterogeneity of gases.

The monomer in a real gas is a basic particle temporarily not entering the effective attraction zones of other particles. Theoretically, the attraction zone is infinite, but it is possible to select the volume, where the attraction potential is deep enough to form the cluster. It is not understandable from microscopic considerations, but the presented here thermodynamics based analysis of experimental thermophysical data permits to find the volume of the cluster effective attraction zone.

The Monomer Fraction Density, $D_{m}$

The monomers' molar concentration is named in this research as the monomer fraction density (MFD), $\mathrm{D}_{\mathrm{m}}$. In zones filled uniquely with monomers the local density is equal to $\mathrm{D}_{\mathrm{m}}$, but in clusters it grows to values inherent to liquids. The heterogeneity of density is reflected also in the local potential energy: it has zero value in the monomer zones, but in clusters it has a large negative value due to attraction of particles to each other. In spite of this heterogeneity, both monomers and basic particles bound in clusters in equilibrium possess the same molar Gibbs energy G, named also as the chemical potential. The Gibbs energy $G$ can be split on the pressure independent part $G_{\text {in }}$ for intramolecular movements and the pressure dependent translational part $\mathrm{G}_{\mathrm{tr}}$.

The monomer fraction is an ideal gas with molar density $\mathrm{D}_{\mathrm{m}}$, and the translational part of the chemical potential $\mathrm{G}_{\text {trm }}$ equals to:

$$
\mathrm{G}_{\mathrm{trm}}=\mathrm{RT} \ln \left(\mathrm{D}_{\mathrm{m}} \mathrm{V}_{\mathrm{q}}\right) \text {. }
$$

Here $\mathrm{V}_{\mathrm{q}}$ is the molar quantum volume for basic particles, proportional to the third power of the de Broglie wavelength, defined by Kittel and Kroemer (1980), as: $\mathrm{V}_{\mathrm{q}}=\mathrm{h}^{3} \mathrm{~N}_{\mathrm{A}}^{4} /(2 \pi \mathrm{MRT})^{3 / 2}$; $\mathrm{h}$ is the Plank constant, $\mathrm{N}_{\mathrm{A}}-$ Avogadro number, $\mathrm{M}$ - molecular mass for basic particles.

In equilibrium the $G_{\text {trm }}$ value keeps for all basic particles, therefore we have:

$$
\mathrm{G}_{\mathrm{tr}}=\mathrm{G}_{\mathrm{trm}} \text {. }
$$

A differentiation of $G_{t r}$ by pressure with account of (2) and (3) gives:

$$
\text { RT } \partial \mathrm{D}_{\mathrm{m}} /\left.\partial \mathrm{P}\right|_{\mathrm{T}}=\mathrm{D}_{\mathrm{m}} / \mathrm{D} \text {. }
$$

The equation (4), derived by Sedunov (2008), looks like the equations, derived by Lewis and Randall (1923), for fugacity and activity. It makes the monomer fraction density relative to these widely used variables in chemical 
physics. But unlike these useful values, which physical interpretation is rather vague, the monomer fraction density has more clear physical sense.

The differential equation (4) permits to find $\mathrm{D}_{\mathrm{m}}\left(\mathrm{P}_{\mathrm{i}}\right)$ at a constant temperature $T$ from regularized experimental set of data $D_{i}\left(T, P_{i}\right)$. The numerical integration of this equation to be correct needs in small steps of the data to be processed and in small initial values for $D_{1}$ and $P_{1}$. The difference between $\mathrm{D}$ and $\mathrm{D}_{\mathrm{m}}$ is clearly seen on the Figure 1 .

Figure 1. The Pressure Dependence of Density D (Thick Line) and the MFD $D_{m}$ (Thin Line) for Carbon Dioxide at $T=400 \mathrm{~K}$

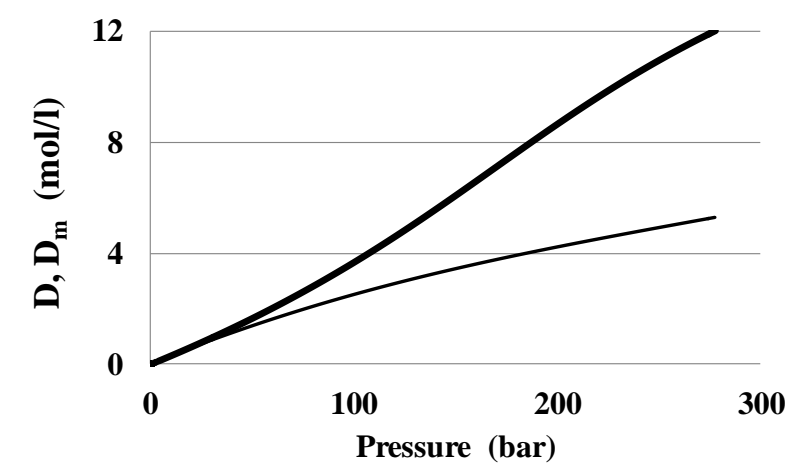

As it is seen from the Figure 1, at small pressures the gas consists mainly of monomers, but at high pressures it is presented mainly by clusters.

So, $\mathrm{D}$ and $\mathrm{D}_{\mathrm{m}}$ are quite different arguments for series expansions of thermophysical values. To follow the Mass Action Law for compound's concentration via concentrations of elementary components, we convert an analyzed molar thermophysical value to its value at a unit volume, in other words - to its density. Then the n-power term of its series expansion by $D_{m}$ will correspond to the contribution of the n-particle cluster fraction in the density of the analyzed value.

The cluster expansion for free energy density of a spin chain by degrees of activity, produced by Bibikov (2014), uses the same basic principles as our method: the argument - activity is relative to our monomer fraction density and the expanded value is the density of energy. Though the spin chains are from an adjacent field of knowledge and stay far from real gases, the basic principles of chemical equilibrium work in the same way for both systems!

It should be noted that the terms of cluster expansions accumulate contributions of different isomers of clusters with the same number of particles. So, the expansion coefficients are combinations of coefficients inherent to isomers, corresponding to the same number of particles. But due to differences between bond parameters of different isomers it becomes possible to select the temperature range, where only one isomer dominates. It permits to estimate the bond parameters inherent to a particular isomer in its zone of domination. 
The Potential Energy Density as the Thermophysical Value Leading to the Clusters' Bond Parameters

Among different thermophysical values of the analyzed gas of prime importance is its potential energy U (T, P), defined by Sedunov (2012b, 2013a) as the difference between the Internal Energy E (T, P) and its zero pressure value $\mathrm{E}(\mathrm{T}, 0)$. The product of $\mathrm{U}$ by $\mathrm{D}$ gives us the potential energy density UD, the series expansion coefficients of which reflect the bond parameters of clusters. We expand in series a positive value $\mathrm{W}=-\mathrm{UD}$. The second order coefficient $\mathrm{W}_{2}$ of the series expansion reflects the properties of the dimer fraction, the higher coefficients $\mathrm{W}_{\mathrm{n}}$ reflect characteristics of n-particle cluster fractions. It should be noted that the n-particle collisions of monomers also contribute to the $\mathrm{W}_{\mathrm{n}}$ value that requires special procedures to clear the clusters' characteristics from the monomer-monomer repulsions. For dimers this problem is solved, and it will be demonstrated in the next subsections.

\section{Extraction of the Cluster Bond Energy from the $W_{n}(T)$ Dependence}

Due to thermal movement the spread of bound states' energy levels in clusters is very high. The cluster bond energy is thermodynamically averaged for all bound states. The cluster bond energy $E_{n}$, defined by Sedunov (2012a) as the cluster dissociation energy, is lower than the depth of the potential well. The $\ln \left(\mathrm{W}_{\mathrm{n}}\right)$ versus $\beta=1 / \mathrm{T}$ plot happens to be linear in the temperature range, where only one isomer dominates, as it was shown by Sedunov (2012a, 2012b, 2013b). This linearity permits to estimate, as the tangent of slope for this part of the graph, the cluster bond energy $E_{n 1}$ in Kelvin, corresponding to the dominating in this range isomer number 1 .

For another linear part of the graph, the $\mathrm{E}_{\mathrm{n} 2}$ value corresponds to the bond energy for another dominating isomer. Linear parts of the graph correspond to ranges of domination of particular isomers. Unlike the abrupt transition between gaseous and liquid phases, the transition between isomer configurations takes a rather wide range of temperatures $\Delta \mathrm{T}$, in which both isomer configurations may coexist. The temperature range of transition between two adjacent linear parts was named by Sedunov (2012a) as the zone of the Soft Structural Transition between dominating isomers in the n-particle cluster fraction. The lower is the difference $\Delta\left(\mathrm{E}_{\mathrm{n}}\right)$ between two bond energies; the larger is the width of the transition zone. In large clusters the difference of bond energies for two dominating isomers may be much larger than T. Then the transition may look like an abrupt first order phase transition.

For changing bond energies Sedunov (2013a) suggested to estimate the $E_{n}$ (T) dependence in $\mathrm{K}$ as the derivative of the $\ln \left(\mathrm{W}_{\mathrm{n}}\right)$ by $\beta$ :

$$
E_{n}(T)=\left(d W_{n} / d \beta\right) / W_{n} \text {. }
$$

As it was noticed earlier, to find the clusters' bond parameters the $\mathrm{W}_{\mathrm{n}}$ values should be cleared from the monomer-monomer repulsions, which influence grows with temperature. For temperatures greatly exceeding the cluster bond energy we use the principle of the cluster bond energy flattening, which requires $\mathrm{E}_{\mathrm{n}}(\mathrm{T})$ to be constant at high temperatures. This principle 
permits to find the temperature dependence for the monomer repulsion contribution to the potential energy density.

\section{Correction of the Pair Bond Energy for Repulsions of Monomers}

At high temperatures there is a nonlinearity of the graph for $\ln \left(\mathrm{W}_{2}\right)$ versus $\beta$, firstly explained by Sedunov (2013b) for atomic gases. The nonlinearity was corrected by an addition to the pair bonding coefficient $\mathrm{W}_{2}$ a small value reflecting the mutual repulsions of two monomers that has proven to grow linearly with temperature for all temperatures reflected in data tables for Noble gases. To estimate the bond energy $E_{2}$ for dimers we use the corrected value $\mathrm{W}_{2 \mathrm{cor}}=\mathrm{W}_{2}+a \mathrm{~T}$ and select the value of $a$ just to flatten the $\mathrm{E}_{2}(\mathrm{~T})$ dependence at high T. The coefficient $a$ can be expressed via an effective volume of repulsions $\mathrm{V}_{\text {rep }}=a / \mathrm{R}$ and changes in the family of Noble gases not very much. The temperature dependence of the repulsion potential energy reflects the form of the pair interaction potential at positive energies of colliding particles.

In molecular gases this phenomenon was also noticed and the coefficient $a$ happened to be in the same range as for atomic gases. It tells that the contribution of mutual repulsions of monomers in the second order potential energy term is not very sensitive to the molecular structure, but reflects the properties of outer electron shells of front atoms responsible for repulsions of colliding molecules. For gases, like Nitrogen, with an enlarged temperature range in the data tables the correction for $\mathrm{W}_{2}$ includes the quadratic term: $\mathrm{W}_{2 \text { cor }}$ $=\mathrm{W}_{2}+a \mathrm{~T}-b \mathrm{~T}^{2}$. The principle of the bond energy flattening at high temperatures permits to find coefficients $a$ and $b$ also in this more complicated case. The division of coefficient $a$ by $b$ gives an estimation of the characteristic temperature $\mathrm{T}_{2}$ for $\mathrm{V}_{\text {rep }}(\mathrm{T})$ dependence: $\mathrm{T}_{2}=a / b$.

For trimers and tetramers the nonlinearity of the $\ln \left(\mathrm{W}_{\mathrm{n}}\right)$ versus $\beta$ graph at high $\mathrm{T}$ is more complicated and requires an additional investigation. This nonlinearity limits the temperature range for determination of the $E_{n}(T)$ values. At moderate temperatures this effect is small and cannot distort the picture of the Soft Structural Transition between cluster isomers.

\section{Determination of the Cluster Fraction Equilibrium Constant}

Dividing $\mathrm{W}_{\mathrm{ncor}}$ by $\mathrm{RE}_{\mathrm{n}}$ we come to the potential energy based equilibrium constant for the $n$-particle cluster fraction: $C_{u n}(T)=W_{n c o r} / R_{n}$. This equilibrium constant accumulates contributions of all isomers of the n-particle cluster fraction. In the Soft Transition Zone it is a combination of partial contributions of all coexisting isomers. It could coincide with the spectroscopically determined equilibrium constant only in the domination range of one isomer, if the spectroscopy might be applied to clusters. The wide spread of bound states' energies for clusters requires a unusual type of spectroscopy. Instead of the bond energy estimation via the spectral peak wavelength, it might be possible to estimate the cluster fractions' characteristics via the dependence of spectroscopic responses on the density of a real gas. 


\section{Determination of the Entropy Factors for Clusters' Bound States}

Dividing the equilibrium constant $\mathrm{C}_{\mathrm{un}}(\mathrm{T})$ by the Boltzmann factor $\exp \left(\mathrm{E}_{\mathrm{n}}\right.$ / $\mathrm{T}$ ) we come to the effective volume $\mathrm{V}_{\mathrm{n}}$ for the cluster attraction zone, which dimension is equal to $3(n-1)$. This effective volume can be considered as the entropy factor for bound states and corresponds to the number of bound states in the cluster $\mathrm{N}_{\mathrm{n}}=\mathrm{V}_{\mathrm{n}} /\left(\mathrm{V}_{\mathrm{q}}\right)^{(\mathrm{n}-1)}$. Unlike chemical compounds with a small number of bound states (the degeneration number of the ground state), the number of bound states in clusters is huge and temperature dependent. But for temperature zones with domination of one isomer the effective volume $V_{n}$ stays constant.

The virial coefficients for $\mathrm{P}(\mathrm{D}, \mathrm{T})$ dependence do not obey to the Boltzmann law. But the Boltzmann law for the potential energy based equilibrium constants of isomer cluster fractions brings clusters closer to chemical compounds and means that the cluster equilibrium constant takes into account all particles in the effective volume $V_{n}$ of the attraction zone: both the bound particles and the particles with positive total energy. For this reason the basic particles with positive total energy in the volume $V_{n}$ are excluded from the monomer fraction.

The cluster bond parameters: bond energies and effective volumes of attraction zones can be extracted from precise experimental thermophysical data for pure gases. The knowledge of bond parameters for small clusters, permits to estimate thermophysical properties of real gases in ranges of density, where dominate small clusters.

\section{The Perspectives of Movement to Larger Densities of Fluids}

To apply the described method to larger clusters we should possess more precise experimental data. But even at a contemporary level of the data precision there are possibilities to estimate some properties of the large cluster fractions. For large clusters in dense gases average numbers of particles in all clusters at definite density and temperature have been estimated by Sedunov (2013c, 2013d) even near critical density at supercritical temperatures. But at densities larger than critical the clusters become substituted by pores in the liquid-like supercritical fluid. It is a challenging task to understand the physics of pores because this knowledge is important for supercritical fluid technologies.

\section{The Bond Parameters for Molecular Gases}

\section{The Bond Parameters for the Nitrogen Real Gas}

The described earlier methods have been used in the computer aided analysis of thermophysical data for Nitrogen real gas. The investigation of this gas is interesting because for Nitrogen the data in the NIST Database are presented in a very wide temperature range, up to $2000 \mathrm{~K}$. The linearity with $\mathrm{T}$ of the monomers' repulsion contribution in $\mathrm{W}_{2}(\mathrm{~T})$ holds for this gas only for $\mathrm{T}$ 
$<800 \mathrm{~K}$. Here we present the results of the $\mathrm{E}_{2}(\mathrm{~T})$ computation for $\mathrm{N}_{2}$ with a quadratic correction for monomers repulsion in the range of temperatures from the triple point $\mathrm{T}_{\mathrm{tr}}=63.2 \mathrm{~K}$ till $2000 \mathrm{~K}$ :

$$
\mathrm{W}_{2 \text { cor }}=\mathrm{W}_{2}+a \mathrm{~T}-b \mathrm{~T}^{2} \text {. }
$$

The correction coefficients for $\mathrm{N}_{2}$ are: $a=0.067$ and $b=1.810^{-6}$.

For comparison, the repulsion coefficient $a$ for Argon equals to 0.047.

The effective repulsion volume for $\mathrm{N}_{2}$ changes with temperature as:

$$
\mathrm{V}_{\text {rep }}=\mathrm{V}_{\text {rep } 0}\left(1-\mathrm{T} / \mathrm{T}_{2}\right) \text {. }
$$

Here $\mathrm{V}_{\text {rep0 }}=0.0081 \mathrm{l} / \mathrm{mol}$ and the nonlinearity factor $\mathrm{T}_{2}=37 \mathrm{kK}$.

The graph for $\ln \left(\mathrm{W}_{2 \mathrm{cor}}\right)$ versus $1000 / \mathrm{T}$ is linear at extremes, Figure 2.

Figure 2. The $\ln \left(W_{2 c o r}\right)$ versus $\beta$ Graph for Nitrogen Real Gas

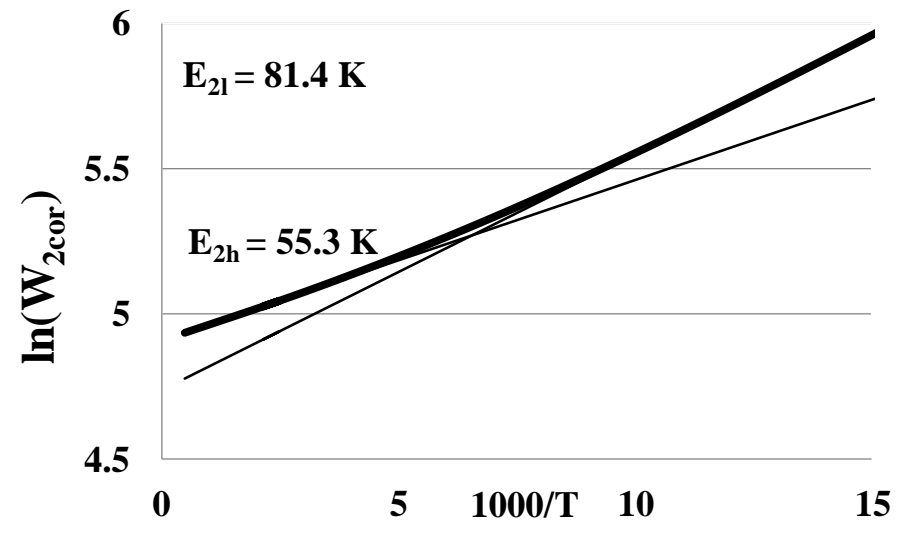

The extreme linear parts of this graph discover the change of the pair bond energy with temperature. At high $\mathrm{T}$ there is a temperature range with almost constant $\mathrm{E}_{2 \mathrm{~h}}(\mathrm{~T})=55.3 \mathrm{~K}$. In this temperature range the $\mathrm{N}_{2}$ molecules move in dimers freely neglecting their angular asymmetry due to high values of the $\mathrm{T} / \mathrm{E}_{2 \mathrm{~h}}$ ratio. Their movement in this temperature range is similar to movements of Noble atoms in dimers at high temperatures. But near the triple point there is a growth of the pair bond energy to $E_{21}=81.4 \mathrm{~K}$ reflecting the angular asymmetry of molecules argued by Gray and Gubbins (1984). The pair bond energy $E_{2}(T)$ for Nitrogen is presented at the Figure 3. 
Figure 3. The Pair Bond Energy $E_{2}(T)$ for Dimers in the Nitrogen Real Gas, Corrected for Monomers' Repulsions

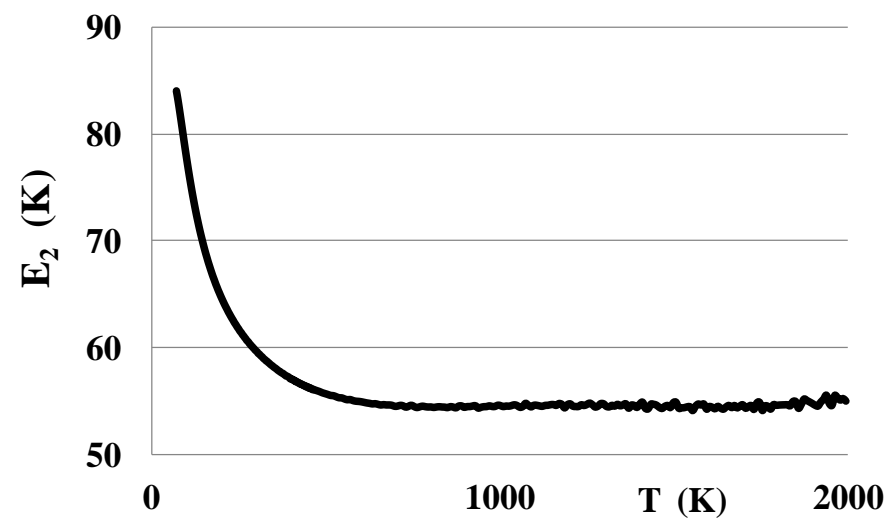

It is seen that the growth of the pair bond energy near the triple point temperature is very large. It tells that the simplified Lennard-Jones model is not applicable to molecular gases because of the mentioned above asymmetry. For this reason the MD computations of thermophysical properties for real molecular gases, based on the Lennard-Jones model, do not provide precision comparable with the precision of regularized experimental data. It may sound strange, but a similar effect had been noticed in Noble gases, thus reflecting the angular asymmetry of their outer electron shells.

\section{The Bond Parameters for Carbon Monoxide Real Gas}

The estimation of the bond energies for two different isomer configurations of the $\mathrm{CO}$ dimers is shown at the Figure 4. Due to low upper limit of temperature the correction for the monomers' repulsion was linear, $a=$ 0.093 . The graph clearly shows the presence of two linear parts with different tangents of slope.

Figure 4. The In $\left(W_{2 c o r}\right)$ versus 1000/T Graph for Dimers in CO Real Gas at Temperatures between the Triple Point $T_{t r}=68.5 \mathrm{~K}$ and $500 \mathrm{~K}$

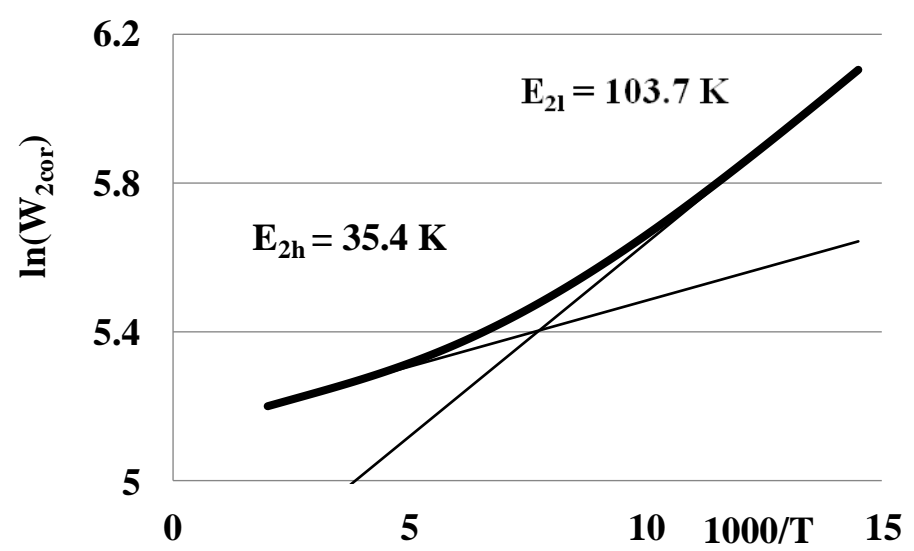


The estimated bond energies for isomers in the dimer fraction are equal: $\mathrm{E}_{21}=103.7 \mathrm{~K}, \mathrm{E}_{2 \mathrm{~h}}=35.4 \mathrm{~K}$. The corresponding values for the bonding volume $\mathrm{V}_{2}$ are: $\mathrm{V}_{21}=0.114 \mathrm{l} / \mathrm{mol}, \mathrm{V}_{2 \mathrm{~h}}=0.576 \mathrm{l} / \mathrm{mol}$. The Soft Transition zone between dominating isomers takes approximately $150 \mathrm{~K}$. At temperatures lower $90 \mathrm{~K}$ the movement of $\mathrm{CO}$ molecules in dimers is performed in a small volume around $0.114 \mathrm{l} / \mathrm{mol}$, but at $\mathrm{T}>240 \mathrm{~K}$ the bonding volume grows five times, to $0.576 \mathrm{l} / \mathrm{mol}$. The diminishing of the bonding volume at low temperatures is the result of the angular dependence of the attraction potential between interacting molecules of CO. At high temperatures the molecules in $\mathrm{CO}$ dimers move freely around each other, neglecting small angular variations of the potential well.

The bond energies for clusters with different numbers of particles in the $\mathrm{CO}$ real gas at temperatures near the triple point are shown at the Figure 5. The linear trends for $\ln \left(\mathrm{W}_{\mathrm{n}}\right)$ versus 1000/T plots give averaged bond energies: for dimers $E_{2}=101 \mathrm{~K}$, for trimers $E_{3}=271 \mathrm{~K}$ and for tetramers $E_{4}=677 \mathrm{~K}$.

The $E_{3}$ to $E_{2}$ ratio in this temperature range is around 2.7. It tells about trimer's structure tending to a closed triangle with 3 pair bonds. For tetramers the ratio $E_{4}$ to $E_{2}$ is around 6.8. It tells about tending to a closed tetrahedral structure of tetramers with 6 pair bonds. So, the computer aided analysis of precise thermophysical data permits to discover the structures of clusters in real gases. It gives directions for the quantum chemistry computations of structures and bond energies for small clusters. It may be most productive at low temperatures, where the thermal movement of molecules is not so influential.

Figure 5. Estimation of Averaged $E_{n}$ for Dimers (Solid Line), Trimers (Triangles), and Tetramers (Squares) in CO Real Gas at T between 68.5 and $120 \mathrm{~K}$

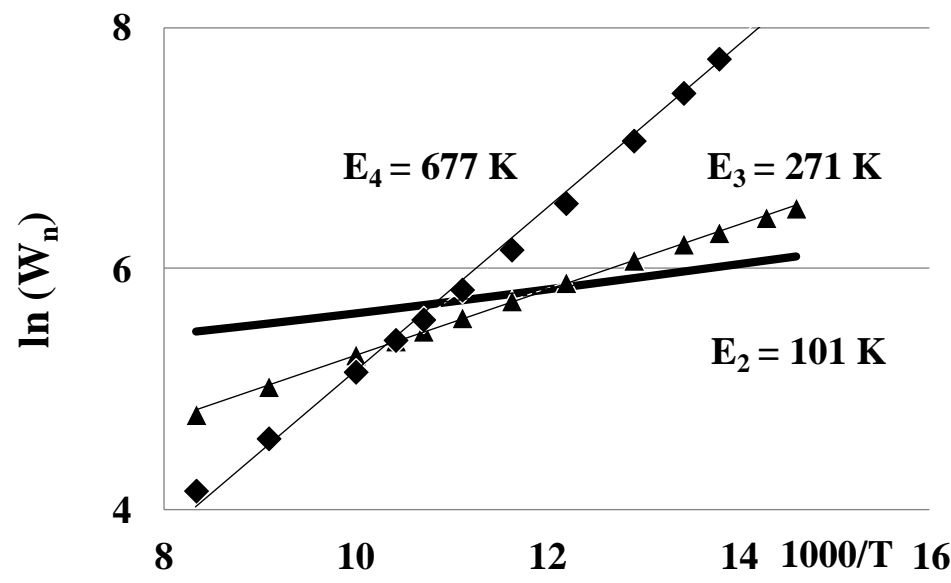

The Pair Bond Energy in the Ammonia Polar Gas

The bond parameters for polar gases, such as ordinary and heavy Water, and Methanol, have been discussed earlier by Sedunov (2013a). In polar gases the cluster bond energies are much larger than in van der Waals gases described in previous subsections. It is seen from the Figure 8, where the pair bond energy is presented for Ammonia. The $\mathrm{W}_{2}(\mathrm{~T})$ data have been corrected 
for monomers' repulsion because of upper temperature reaching $700 \mathrm{~K}$. The shown here change of the bond energy with temperature may help in investigation of the Hydrogen bonds' nature. The monomers repulsion correction coefficient $a$ in this case is equal to 0.08 and the corresponding value for the effective repulsion volume is $0.0096 \mathrm{l} / \mathrm{mol}$.

Figure 8. The Temperature Dependence of the Pair Bond Energy $E_{2}(T)$ for Ammonia Polar Gas

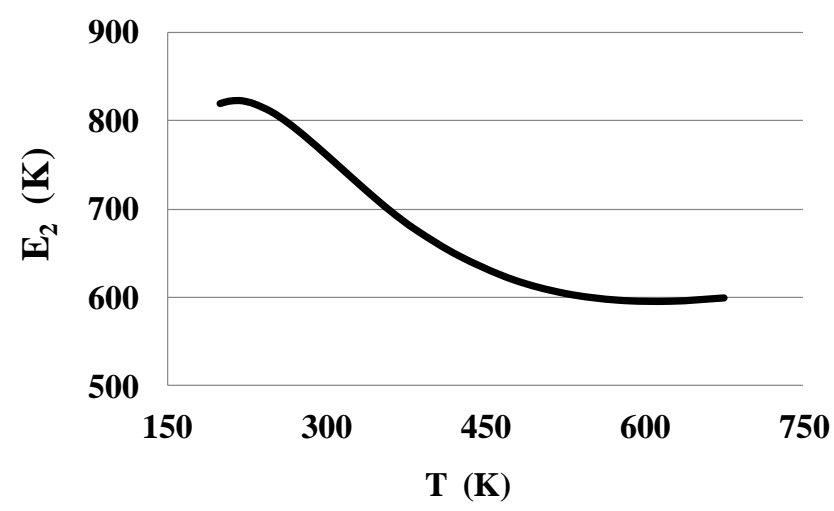

It is seen that at low temperatures the bond energy reaches its maximal value and even slightly falls to lower temperatures. The fall of the pair interaction bond energy at low temperatures was noticed by Sedunov (2013a) for different Hydrogen containing gases, including Hydrocarbons. This effect is very important and requires a further investigation.

\section{The Discussion of Results}

The Accounting for the Monomer Repulsion in Computations of the Clusters' Bond Parameters

All three examples of the dimer bond energy computation for quite different gases show an effectiveness of clearing the second coefficient for dimer potential energy density expansion from the two-particle collision energy. All graphs for $\mathrm{E}_{2}(\mathrm{~T})$ become flattened after this correction. This correction is a key factor of the method permitting to utilize effectively the data from the NIST Database in all temperature range. The flattening at high $\mathrm{T}$ of the $E_{2}(T)$ is quite natural and understandable. A similar flattening should take place for larger clusters, but its mechanism is more complicated and needs an additional investigation.

\section{The Temperature Dependence of the Dimer Bond Energy}

The demonstration of the dimer bond energy essential growth at low temperatures confirms the conclusions of Gray and Gubbins (1984) about the role of the molecular interaction potential angular dependence. It motivates the dimer bond energy investigations at different forms of potential. It should be 
noted that the molecular interactions potential is temperature independent. The Soft Structural Transition in cluster fractions results from the redistribution of molecules in the potential well. At low temperatures molecules dive in the potential well, thus diminishing own effective volume of the attraction zone. At higher temperatures, due to the growing influence of the entropy factor, the effective volume of the attraction zone expands, thus diminishing the average bond energy.

\section{Estimation of Averaged Bond Energies in Different Clusters}

At moderate temperatures, where the monomer repulsions are not very influential, the comparison of the bond energies for dimers, trimers and tetramers permits to estimate the number of pair bonds in trimers and tetramers, thus providing information about their possible configuration. It is interesting to find confirmation for the resulting cluster structure from the quantum chemistry computations.

\section{Conclusions}

- The monomer fraction density, as the main variable in the computerized thermal analysis of fluids, is a key to the structure of fluids.

- The potential energy based analysis of fluids' thermophysical properties discovers the cluster structure of equilibrium fluids.

- The regularized experimental data in modern electronic databases of thermophysical properties possess high enough precision for the determination of the molecular interaction and cluster bond parameters.

- The bond parameters for isomers of equilibrium clusters can be determined in temperature zones of their domination.

- The equilibrium constants for dominating isomers of clusters in real gases, found on the potential energy basis, obey to the Boltzmann law.

- The theory of small cluster fractions in diluted gases creates a foundation for further movement to larger clusters and larger densities.

- The found and anticipated results inspire and provide directions for more profound experimental and theoretical studies of real gases and condensed matter.

\section{Nomenclature}

D Molar density of a real gas, $(\mathrm{mol} / \mathrm{l})$

$\mathrm{D}_{\mathrm{m}} \quad$ Monomer fraction density, $(\mathrm{mol} / \mathrm{l})$

P Pressure, (bar) 
$\mathrm{T} \quad$ Equilibrium temperature, $(\mathrm{K})$

$\beta \quad$ The reverse temperature, $(1 / \mathrm{K})$

E Molar Internal energy, $(\mathrm{J} / \mathrm{mol})$

G Molar Gibbs free energy, Chemical potential, $(\mathrm{J} / \mathrm{mol})$

$\mathrm{G}_{\text {tr }} \quad$ Translational part of the Gibbs energy, $(\mathrm{J} / \mathrm{mol})$

$\mathrm{G}_{\text {int }} \quad$ Intramolecular part of the Gibbs energy, $(\mathrm{J} / \mathrm{mol})$

$\mathrm{U} \quad$ Molar potential energy of a gas, $(\mathrm{J} / \mathrm{mol})$

W Potential energy density, $(\mathrm{J} / \mathrm{l})$

$\mathrm{V}_{\mathrm{q}} \quad$ Molar quantum volume for basic particles, $(\mathrm{l} / \mathrm{mol})$

h Plank's constant

M Molecular weight, $(\mathrm{kg} / \mathrm{mol})$

$\mathrm{N}_{\mathrm{A}} \quad$ Avogadro number, $(1 / \mathrm{mol})$

$\mathrm{R} \quad$ Universal gas constant, $\mathrm{J} /(\mathrm{mol} \mathrm{K})$

$\mathrm{R}_{\mathrm{ef}} \quad$ Zero pressure limit of P/TD ratio for an analyzed data table, $\mathrm{J} /(\mathrm{mol} \mathrm{K})$

$\mathrm{n} \quad$ Number of particles in the cluster and the order of the series expansion term

$\mathrm{W}_{\mathrm{n}} \quad \mathrm{n}$-order series expansion coefficient for $\mathrm{W},\left(\mathrm{J}^{\mathrm{n}-1} / \mathrm{mol}^{\mathrm{n})}\right.$

$\mathrm{W}_{2 \mathrm{cor}}$ The dimer expansion coefficient corrected for the monomer repulsion

$\mathrm{E}_{\mathrm{n}}(\mathrm{T})$ Cluster bond energy, $(\mathrm{K})$

$\mathrm{C}_{\mathrm{un}}(\mathrm{T})$ Equilibrium n-order cluster formation constant based on the potential energy density series expansion, $(1 / \mathrm{mol})^{\mathrm{n}-1}$

$\mathrm{V}_{\mathrm{n}} \quad$ Molar volume of the cluster effective attraction zone, $(1 / \mathrm{mol})^{\mathrm{n}-1}$

$a \quad$ The coefficient for linear temperature correction of $\mathrm{W}_{2}(\mathrm{~T})$

$b \quad$ The coefficient for quadratic temperature correction of $\mathrm{W}_{2}(\mathrm{~T})$

\section{References}

Aster, R.C., Borchers, B. and Thurber, C. (2012) Parameter Estimation and Inverse Problems, 2nd Ed. Amsterdam: Elsevier.

Bibikov, P.N. (2014) Second cluster integral from the spectrum of an infinite XXZ spin chain, [Online] Available from: arXiv.org/pdf/1408.4586 [Accessed: $20^{\text {th }}$ December 2014].

Economou, I.G. (2002) Statistical Associating Fluid Theory: A Successful Model for Calculation of Thermodynamic and Phase Equilibrium Properties of Complex Fluid Mixtures, Ind. Eng. Chem. Res., 41 p. 953-962.

Feynman, R. (1972) Statistical mechanics. A set of lectures, Massachusetts: W.A. Benjamin, Inc.

Gray, C.G. and Gubbins, K.E. (2011) Theory of Molecular Fluids, in 2 volumes. Oxford NY: Oxford University Press.

Kaplan, I.G. (2006) Intermolecular Interactions: Physical Picture, Computational Methods and Model Potentials, Hoboken, NJ: John Wiley \& Sons.

Kittel, Ch. and Kroemer, H. (1980) Thermal physics, 2nd Ed. New York: W.H. Freeman and Co.

Kontogeorgis, G.M. and Folas, G. K. (2009) Thermodynamic models for industrial applications: from classical and advanced mixing rules to association theories, Hoboken, NJ: John Wiley \& Sons

Koudryavtsev, A.B., Jameson, R.F. and Linert, W. (2001) The Law of Mass Action, Berlin: Springer-Verlag. 
Landau, L.D., Lifshits, E.M. (1980) Statistical physics, London: Pergamon Press.

Lennard-Jones, J. E. (1924) On the determination of molecular fields from the equation of state of a gas, Proceedings of the Royal Society A, 106 (738) p. 441462.

Lewis, G. N. and Randall, M. (1923) Thermodynamics and the Free Energy of Chemical Substances, New York: McGraw-Hill.

Mayer, J.E. and Goeppert-Mayer, M. (1977) Statistical Mechanics, 2nd Ed. New York: John Wiley \& Sons.

Poling, B.E., Prausnitz, J.M. and O'CONNELL, J. P. (2001) The Properties of Gases and Liquids. 5th Ed. New York: McGraw-Hill.

Rowlinson, J.S. (2002) Cohesion: A Scientific History of Intermolecular Forces, Cambridge: University Press.

Sedunov, B. (2008) Monomer fraction in real gases. Int. J. of Thermodynamics, 11 (1) p. 1-9.

Sedunov, B. (2012a) Equilibrium molecular interactions in pure gases. J. of Thermodynamics, [Online] Available from: http://dx.doi.org/10.1155/2012/ 859047 [Accessed: $25^{\text {th }}$ May 2014].

Sedunov, B. (2012b) The Analysis of the Equilibrium Cluster Structure in Supercritical Carbon Dioxide, American J. of Analytical Chemistry, 3 (12A) p. 899-904.

Sedunov, B. (2013a) Thermal Analysis of Thermophysical Data for Equilibrium Pure Fluids, J. of Modern Physics, Special issue: Thermal Analysis Methods 4 (7B), p. 8-15.

Sedunov, B. (2013b) Equilibrium Thermal Physics of Noble Gases J. of Modern Physics, 4 (12B) p. 8-14.

Sedunov, B. (2013c) Equilibrium Structure of Dense Gases, Proc. of the JEEP-2013, Nancy, MATEC Web of Conferences [Online] Available from: http://dx.doi. org/10.1051/matecconf/20130301002 [Accessed: $16^{\text {th }}$ June 2014].

Sedunov, B. (2013d) The Equilibrium Thermal Physics of Supercritical Fluids, Int. J. of Analytical Mass Spectrometry and Chromatography, 1 (2) p. 103-108.

TRC.NIST (2014) Thermodynamics Research Center. [Online] Available from: http:// trc.nist.gov/ [Accessed: 14 ${ }^{\text {th }}$ May 2014].

WEBBOOK.NIST (2011) Thermophysical Properties of Fluid Systems. [Online] Available from: http://webbook.nist.gov/chemistry/fluid/ [Accessed: $14^{\text {th }}$ May 2014]. 
